\title{
Explosion and nucleosynthesis of low-redshift pair-instability supernovae ${ }^{\star}$
}

\author{
A. Kozyreva ${ }^{1}$, S.-C. Yoon ${ }^{1,2}$, and N. Langer ${ }^{1}$ \\ 1 Argelander-Institut für Astronomie, Universität Bonn, Auf dem Hügel 71, 53121 Bonn, Germany \\ e-mail: kozyreva@astro.uni-bonn.de \\ 2 Astronomy Program, Department of Physics \& Astronomy, Seoul National University, 151-742 Seoul, Republic of Korea \\ Received 14 February 2014 / Accepted 24 May 2014
}

\section{ABSTRACT}

\begin{abstract}
Context. Both recent observations and stellar evolution models suggest that pair-instability supernovae (PISNe) could occur in the local Universe, at metallicities below $\lesssim Z_{\odot} / 3$. Previous PISN models were mostly produced at very low metallicities in the context of the early Universe.

Aims. We present new PISNe models at a metallicity of $Z=0.001$, which are relevant for the local Universe.

Methods. We took previously published self-consistent stellar evolutionary models of pair-instability progenitors with initial masses of $150 M_{\odot}$ and $250 M_{\odot}$ at metallicity of $Z=0.001$ and followed the evolution of these models through the supernova explosions, using a hydrodynamics stellar evolution code with an extensive nuclear network including 200 isotopes.

Results. In both models the stars explode as PISNe without leaving a compact stellar remnant. Our models produce a nucleosynthetic pattern that is generally similar to that of Population III PISN models, which is mainly characterized by the production of large amounts of $\alpha$-elements and a strong deficiency of the odd-charged elements. However, the odd-even effect in our models is significantly weaker than that found in Population III models. The comparison with the nucleosynthetic yields from core-collapse supernovae at a similar metallicity $(Z=0.002)$ indicates that PISNe could have strongly influenced the chemical evolution below $Z \approx 0.002$, assuming a standard initial mass function. The odd-even effect is predicted to be most prominent for the intermediatemass elements between silicon and calcium.

Conclusions. With future observations of chemical abundances in Population II stars, our result can be used to constrain the number of PISNe that occurred during the past evolution of our Galaxy.
\end{abstract}

Key words. nuclear reactions, nucleosynthesis, abundances - stars: evolution - stars: massive - supernovae: general stars: abundances

\section{Introduction}

The pair-instability mechanism for supernova explosions was first suggested in the 1960s (Fowler \& Hoyle 1964; BisnovatyiKogan \& Kazhdan 1967; Rakavy \& Shaviv 1967; Barkat et al. 1967; Fraley 1968; Zeldovich \& Novikov 1971). The cores of very massive stars with initial masses higher than about $100 M_{\odot}$ (Bond et al. 1982; Heger et al. 2003) have relatively low densities and high temperatures for which radiation pressure is dominant over gas pressure. When the core temperature approaches $10^{9} \mathrm{~K}$, the creation of electron-positron pairs out of gamma-ray photons from the high-energy tail of the blackbody spectrum becomes important and causes the adiabatic in$\operatorname{dex} \Gamma$ drop below $4 / 3$. This in turn causes gravitational collapse of the core if a significant fraction of the core has $\Gamma<4 / 3$. The consequent oxygen burning induces a thermonuclear explosion that completely disrupts the star if the released energy exceeds its binding energy. This occurs for oxygen core masses above approximately $45 M_{\odot}$.

For a pair-instability supernova to occur, its progenitor needs to retain a high enough mass to keep its helium core mass above about $\sim 65 M_{\odot}$. This condition cannot be easily fulfilled at high metallicity, for which the evolution of very massive stars are dominated by stellar wind mass-loss (e.g. Vink et al. 2011).

\footnotetext{
* Table 3 is also available in electronic form at the CDS via anonymous ftp to cdsarc.u-strasbg. fr (130.79.128.5) or via http://cdsarc.u-strasbg.fr/viz-bin/qcat?J/A+A/566/A146
}

This is the reason why most theoretical studies of pair-instability supernovae (PISNe) have focused on zero or extremely metalpoor stars in the early Universe (El Eid et al. 1983; Umeda \& Nomoto 2002; Heger \& Woosley 2002; Scannapieco et al. 2005; Kasen et al. 2011; Pan et al. 2012a; Whalen et al. 2013a; Dessart et al. 2013). However, Langer et al. (2007) recently pointed out that the metallicity threshold for PISNe can be as high as $Z_{\odot} / 3$ within the current theoretical uncertainty of stellar wind massloss rates, implying one PISN per one thousand supernovae in the local Universe.

Pair-instabilitly SNe would be marked by broad light curves given their high progenitor masses. They would also appear extremely luminous if their progenitors have large radii and/or if a large amount of nickel is produced as a result of the pair-creation instability (e.g., Scannapieco et al. 2005; Kasen et al. 2011). This raises the question whether some of the super-luminous $\mathrm{SNe}$ of various types, such as SN 2006gy and SN 2007bi discovered in the nearby Universe, have a pair-instability origin (see Gal-Yam 2012, for a review). For example, the light curve of SN 2007bi implies the radioactive decay of more than $3 M_{\odot}$ of nickel, for which a pair-instability explosion gives one of the best explanations (Gal-Yam et al. 2009). Alternative possibilities are supernovae powered by a young magnetar, as suggested by various authors (Kasen \& Bildsten 2010; Dessart et al. 2012), and interaction-driven supernova (Moriya et al. 2010). If local PISNe would exist, one would have to wonder how they would have impacted on the chemical evolution of the local Universe. 
Table 1. Properties of our PISN progenitor models and of similar Population III helium star models from Heger \& Woosley (2002).

\begin{tabular}{lccccccc}
\hline \hline Initial mass & $\begin{array}{c}\text { Final mass } \\
{\left[M_{\odot}\right]}\end{array}$ & $\begin{array}{c}\text { He-core } \\
{\left[M_{\odot}\right]}\end{array}$ & $\begin{array}{c}\text { O-core } \\
{\left[M_{\odot}\right]}\end{array}$ & $\begin{array}{c}T_{\mathrm{c}}^{\max } \\
{\left[10^{9} \mathrm{~K}\right]}\end{array}$ & $\begin{array}{c}\log \rho_{\mathrm{c}}^{\max } \\
{\left[\mathrm{g} \mathrm{cm}^{-3}\right]}\end{array}$ & $\eta_{\mathrm{c}}^{\text {init }}$ & $\eta_{\mathrm{c}}^{\max }$ \\
\hline $150 M_{\odot}$ & 94 & 72 & 64 & 3.45 & 6.25 & $1.0 \times 10^{-4}$ & $2.5 \times 10^{-4}$ \\
$70 M_{\odot} \mathrm{He}$ & 70 & 70 & 60 & 3.57 & 6.30 & $1.9 \times 10^{-7}$ & $2.8 \times 10^{-4}$ \\
\hline $250 M_{\odot}$ & 169 & 121 & 110 & 5.12 & 6.69 & $1.0 \times 10^{-4}$ & $1.6 \times 10^{-3}$ \\
$115 M_{\odot} \mathrm{He}$ & 115 & 115 & 90 & 5.14 & 6.67 & $1.9 \times 10^{-7}$ & $7.3 \times 10^{-4}$ \\
\hline
\end{tabular}

Notes. $T_{\mathrm{c}}^{\max }$ and $\rho_{\mathrm{c}}^{\max }$ are the highest central temperature and central density that are achieved during the calculations. The last two columns give the values of the central neutron excess initially and at the highest temperature.

Addressing these questions requires PISN models that are relevant to the environment of the local Universe. The first studies of local PISN models were performed by Langer \& El Eid (1986), El Eid \& Langer (1986), and Herzig et al. (1990), who calculated evolutionary models with an initial mass of $100 M_{\odot}$ and a metallicity of $Z=0.03$. More recently, Langer et al. (2007) calculated $150 M_{\odot}$ and $250 M_{\odot}$ models at a metallicity of $Z=0.001$ as PISN progenitors, adopting the most up-todate prescriptions for stellar wind mass-loss rates. These models provide self-consistent progenitor models for PISNe in the local Universe together with more recent models by Yusof et al. (2013). In the present study we follow the evolution of two models from Langer et al. (2007) through the explosive oxygen and silicon burning stages to verify that they explode via the pair-instability mechanism, and to discuss implications for nucleosynthesis in the local Universe. Their shock-breakout signatures and light curves will be discussed in a separate paper (Kozyreva et al. 2014).

This paper is organized as follows. We describe the numerical method we adopted in Sect. 2. The results of our calculations are reported in Sect. 3, where we also discuss the nucleosynthesis yields of our PISN models. We discuss the implications of our results for the chemical evolution of the local Universe in Sect. 4, and conclude our study in the final section.

\section{Numerical method and input physics}

We used an implicit Lagrangian hydrodynamics code that solves the difference equations for the stellar structure iteratively by the Henyey relaxation method (Henyey et al. 1964; Heger et al. 2000; Yoon \& Langer 2005; Yoon et al. 2006). We list the relevant stellar structure equations in Appendix A. The equation of state is based on Blinnikov et al. (1996) and includes ions, electrons and positrons, radiation, degeneracy effects, and ionization contributions. The opacity was computed from the OPAL tables (Iglesias \& Rogers 1996) and Alexander \& Ferguson (1994).

We computed the nucleosynthesis and the corresponding energy generation rate in the following way: for temperatures lower than $4.5 \times 10^{8} \mathrm{~K}$, a small nuclear network (39 isotopes) was used. For higher temperatures we used the "torch" nuclear network developed by Timmes $(1998,1999)$ with 200 isotopes. In this network, the weak interactions are followed using the data provided by Fuller et al. (1982). For a temperature range where silicon burning is well described in terms of quasi-statistical equilibrium (QSE, Bodansky et al. 1968; Hix \& Thielemann 1996), energy generation rates can be given as a function of temperature $T$, density $\rho$, total mass fraction of the silicon QSE-group ${ }^{1}$ elements $X_{\mathrm{Si}}$ and electron abundance $Y_{\mathrm{e}}$. To

\footnotetext{
1 QSE groups are the groups of isotopes formed in the condition of quasi-statistical equilibrium (Wallerstein et al. 1997).
}

calculate the energy generation rates during silicon burning, we therefore used an energy generation rate table for a number of combinations of different physical parameters $(T=(2.4-5) \times$ $\left.10^{9} \mathrm{~K}, \log _{10}\left(\rho /\left(\mathrm{g} \mathrm{cm}^{-3}\right)\right)=5-10, X_{\mathrm{Si}}=0-1, Y_{e}=0.44-0.5\right)$, following Nomoto \& Hashimoto (1988). For very high temperatures $\left(T>5 \times 10^{9} \mathrm{~K}\right)$, the nuclear statistical equilibrium routine by Timmes (1998) was employed.

Our starting models are taken from the stellar evolutionary calculations with initial masses of $150 M_{\odot}$ and $250 M_{\odot}$ and an initial rotational velocity of $10 \mathrm{~km} \mathrm{~s}^{-1}$ at $Z=0.001$ by Langer et al. (2007, the model sequences 3 and 4 ). These models were calculated from the zero-age main sequence until the onset of the pair instability in the core, with the stellar wind massloss prescription described in Yoon et al. (2006). The starting point of our calculations is core carbon exhaustion, after which these stars quickly enter the pair instability phase. The stellar masses at this point are $94 M_{\odot}$ and $169 M_{\odot}$ for the $150 M_{\odot}$ and $250 M_{\odot}$ stars, respectively. We summarize some model properties in Table 1 along with those of zero-metallicity models by Heger \& Woosley (2002) for comparison.

The stellar evolutionary models from Langer et al. (2007) were calculated using the Ledoux criterion for convection, with the assumption of semi-convection (Langer et al. 1983) using a large semi-convective mixing parameter $\left(\alpha_{\mathrm{SEM}}=1\right.$, Langer 1991), and without convective-core overshooting. The mixing length parameter was chosen to be 1.5 of pressure scale height (Yoon et al. 2006). We neglected the convective mixing during the explosive oxygen and silicon-burning phases in our calculations because the convection timescale is two orders of magnitude larger than the hydrodynamical timescale on which the collapse induced by the pair instability develops.

Note that the recent PISN progenitor models at higher metallicity (0.002 and 0.006) by Yusof et al. (2013) were computed using the Schwarzschild criterion for convection and with core overshooting with a moderate overshooting parameter $\left(\alpha_{\text {over }}=0.1\right)$. The convection in the outer layers was calculated with the mixing-length parameter scaled to the density scale height ( $\left.\alpha_{\mathrm{MLT}}=l / H_{\rho}=1\right)$ to avoid density inversions (see also Ekström et al. 2012). The consequences of this treatment are more compact stellar models, a lower degree of mass loss, and larger carbon-oxygen cores. The models from Yusof et al. (2013), which are claimed to produce PISNe, were evolved until the end of helium/oxygen burning. Electron-positron pair creation was not included in the equation of state of the employed evolutionary code. The statement about the PISN fate is based on the size of the carbon-oxygen core. In a more recent study the PISN models from Yusof et al. (2013) (at the end of core helium burning) were mapped into the KEPLER code (Heger \& Woosley 2010). With these calculations the models were evolved through pair instability and eventually exploded (Whalen et al. 2013b). 
A. Kozyreva et al.: Explosion and nucleosynthesis of low-redshift pair-instability supernovae

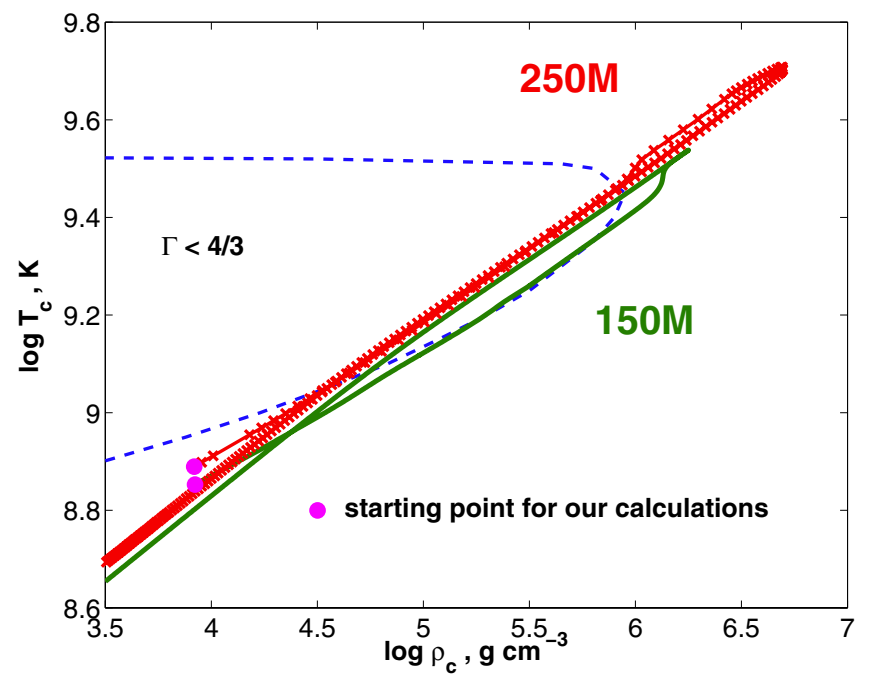

Fig. 1. Evolutionary tracks of our $150 M_{\odot}$ (labelled "150M", solid line) and $250 M_{\odot}$ (labelled "250M", line with time signs) models in central density - temperature diagram. The area enclosed by the dashed line indicates the pair-instability regime where $\Gamma<4 / 3$. The filled circles mark the starting points for each model sequence.

Rotation is not included in our calculations because these models retain very little angular momentum.

\section{Results}

\subsection{Explosion}

Through the hydrodynamics terms included in the BEC code (see Appendix A) we followed the dynamical phase of the evolution of our PISN models. Usually, hydrodynamic stellar evolution codes are not able to describe dynamical processes in stars because of the implicit nature of the adopted numerical solvers (which cause strong numerical damping) and the long time steps that are required to follow the evolution timescale (Appenzeller 1970; Woosley \& Weaver 1982). Pulsations and shock waves can be resolved, however, if the time step becomes similar to the dynamical characteristic time (possible during late stages of stellar evolution), and if the growth rate of a hydrodynamical phenomenon is sufficiently high (El Eid \& Langer 1986; Heger et al. 1997; Yoon \& Cantiello 2010).

We find that both models explode as a result of explosive nuclear burning during the pair-instability phase, which confirms the prediction by Langer et al. (2007).

In Fig. 1, the evolutionary tracks of the central density and temperature are shown. Both quantities increase rapidly during the dynamical contraction of the core induced by the pair instability. The highest temperature and density achieved during this phase are $T_{\mathrm{c}}=3.45 \times 10^{9} \mathrm{~K}$ and $\rho_{\mathrm{c}}=1.8 \times 10^{6} \mathrm{~g} \mathrm{~cm}^{-3}$ for the $150 M_{\odot}$ star, and $T_{\mathrm{c}}=5.1 \times 10^{9} \mathrm{~K}$ and $\rho_{\mathrm{c}}=4.9 \times 10^{6} \mathrm{~g} \mathrm{~cm}^{-3}$ for the $250 M_{\odot}$ star. As shown in Table 1, these values are similar to those found in Population III star models by Heger \& Woosley (2002) for similar helium core masses. Beyond this point, the contraction is reversed, and the star explodes.

Figure 2 illustrates that our models explode because of the nuclear energy release. Initially, our models have a negative binding energy $\left(E_{\text {bind }}=E_{\text {grav }}+E_{\text {thermal }}\right)$. The pair creation triggers the collapse, which is visible as a minor increase of the kinetic energy around $t=0$ for model $150 \mathrm{M}$ and around $t=0-10 \mathrm{~s}$ for model $250 \mathrm{M}$. The consequent oxygen and silicon burning occur with a sharp decrease of the nuclear binding energy.
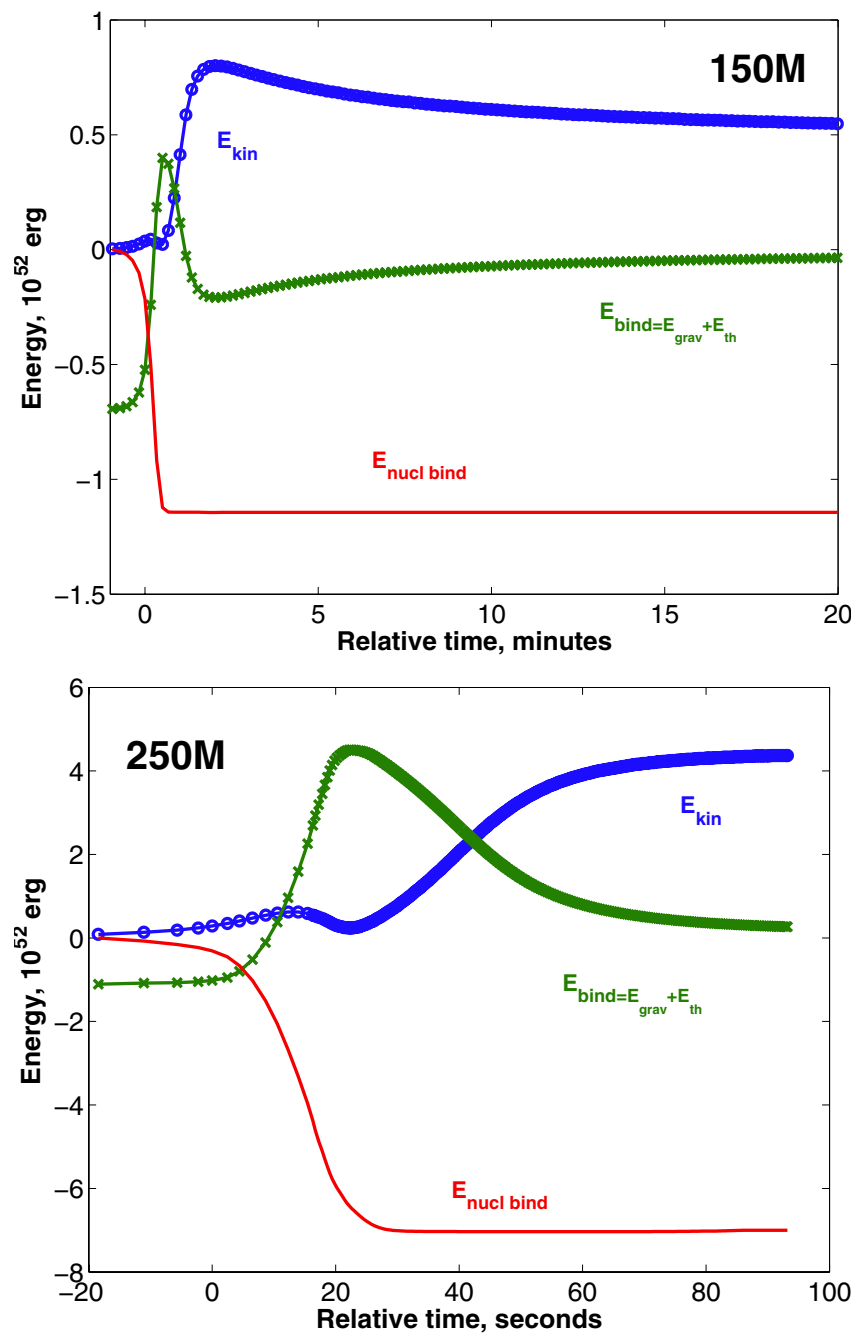

Fig. 2. Evolution of the energetics for our $150 M_{\odot}$ and $250 M_{\odot}$ models. We show the kinetic energy (blue, circles), the binding energy (green, times) that is the sum of gravitational and thermal energies, and the nuclear binding energy (red) of the stars. Here, the nuclear binding energy is defined by the difference between the total nuclear binding energy of all nuclei at the end of carbon burning and that of a given evolutionary epoch (see description in the text). The zero point in time is defined as the time of the beginning of pair-instability explosion.

The nuclear binding energy is defined the following way:

$E_{\mathrm{bind}}=\sum_{k} \sum_{i} X_{i} e_{i} \frac{\Delta M_{k}}{m_{i}}$,

where $X_{i}$ represents the mass fractions of the isotopes, $e_{i}$ and $m_{i}$ are the nuclear binding energy and nuclear mass of the isotope $i$, and $\Delta M$ is the mass of the mass shell of the numerical stellar model. The sum is made over all mass shells and over all isotopes. Figure 2 shows the difference of nuclear binding energy at a given time to that at the end of carbon burning, that is, the first time point in the figures. The drop in the nuclear binding energy shows the amount of energy released by nuclear burning during the pair-instability explosion.

The released nuclear energy from oxygen and silicon burning is converted into thermal and kinetic energy, resulting in a positive binding energy. A positive binding energy means that the system becomes unbound. Eventually, most of the released nuclear energy is converted into kinetic energy, which is strong evidence for the explosion of the star. The final kinetic energy 
Table 2. Total nucleosynthetic yields for selected isotopes in solar masses for our $150 M_{\odot}$ and $250 M_{\odot}$ models (including matter lost by stellar wind and decay products) in comparison with $70 M_{\odot}$ and $115 M_{\odot}$ zero metallicity helium star yields (Heger \& Woosley 2002), respectively.

\begin{tabular}{|c|c|c|c|c|c|c|c|c|c|c|c|c|c|c|}
\hline & $Z$ & ${ }^{1} \mathrm{H}$ & ${ }^{4} \mathrm{He}$ & ${ }^{12} \mathrm{C}$ & ${ }^{16} \mathrm{O}$ & ${ }^{20} \mathrm{Ne}$ & ${ }^{24} \mathrm{Mg}$ & ${ }^{28} \mathrm{Si}$ & ${ }^{32} \mathrm{~S}$ & ${ }^{36} \mathrm{Ar}$ & ${ }^{40} \mathrm{Ca}$ & ${ }^{46} \mathrm{Ti}$ & ${ }^{50} \mathrm{Cr}$ & ${ }^{56} \mathrm{Fe}$ \\
\hline $150 M_{\odot}$ & 0.001 & $36.6 / 4.9$ & $49.2 / 24.4$ & 2.2 & 46.9 & 2.6 & 2.3 & 6.2 & 2.8 & 0.5 & 0.5 & $10^{-4}$ & $10^{-4}$ & 0.04 \\
\hline He $70 M_{\odot}$ & 0 & - & 1.5 & 4.5 & 45.8 & 4.0 & 3.0 & 8.0 & 2.4 & 0.3 & 0.2 & $10^{-4}$ & $10^{-4}$ & 0.01 \\
\hline $250 M_{\odot}$ & 0.001 & $57.1 / 10.3$ & $81.5 / 47.5$ & 0.9 & 42. & 1.8 & 2.5 & 23.1 & 14.3 & 2.9 & 2.8 & $10^{-4}$ & $10^{-4}$ & 19.4 \\
\hline He $115 M_{\odot}$ & 0 & - & 1.8 & 3.7 & 40. & 3.8 & 4.5 & 25.7 & 11.8 & 1.9 & 1.6 & $10^{-5}$ & $10^{-4}$ & 19.0 \\
\hline
\end{tabular}

Notes. Yields for hydrogen and helium neglecting the wind matter are given after the slash sign.
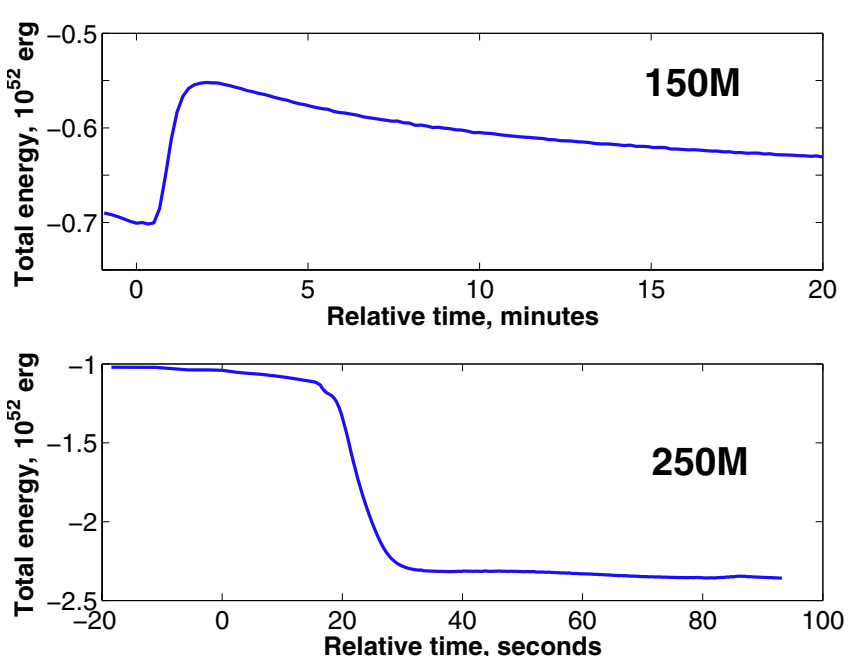

Fig. 3. Total energy evolution for our $1500 M_{\odot}$ (upper) and $250 M_{\odot}$ (bottom) models.

is 8 foe $^{2}$ for model $150 \mathrm{M}$ and 44 foe for model $250 \mathrm{M}$. This corresponds to an asymptotic velocity of the ejecta at the infinity of $2.9 \times 10^{3} \mathrm{~km} \mathrm{~s}^{-1}$ for model $150 \mathrm{M}$ and $5.1 \times 10^{3} \mathrm{~km} \mathrm{~s}^{-1}$ for model 250M.

The BEC code has no provision for treating shocks. However, because of the strong density contrast at the base of the hydrogen-rich envelope, a shock wave develops at this point due to the explosion of the carbon-oxygen core. Its Mach number is about 2 for both our models. As a consequence, energy is not perfectly conserved in our models at the time when the shock enters the stellar envelope (see Fig. 3). Still, the total energy is conserved to better than $5 \%$ in our $150 M_{\odot}$ model, and to better than $20 \%$ in our $250 M_{\odot}$ model. We note that the velocities scale with $\sqrt{E_{\text {kin }}}$, and that we expect our velocities to be precise to $3 \%$ and $10 \%$, respectively. The comparison of Figs. 2 and 3 shows that energy conservation during the nuclear burning phase is very good, such that our nucleosynthesis results are not affected by this problem.

\subsection{Nucleosynthesis}

Figure 4 shows the final chemical structure of our models. The total amounts of produced ${ }^{56} \mathrm{Ni}$ are $0.04 M_{\odot}$ and $19.3 M_{\odot}$ for the $150 M_{\odot}$ and $250 M_{\odot}$ star, respectively. As shown in Table 2, the overall nucleosynthetic results agree well with that of the $70 M_{\odot}$ and $115 M_{\odot}$ Population III helium star models by Heger \& Woosley (2002), whose masses are similar to the He core masses of our models.

\footnotetext{
21 foe $=10^{51} \mathrm{erg}$ (from "fifty-one-erg").
}
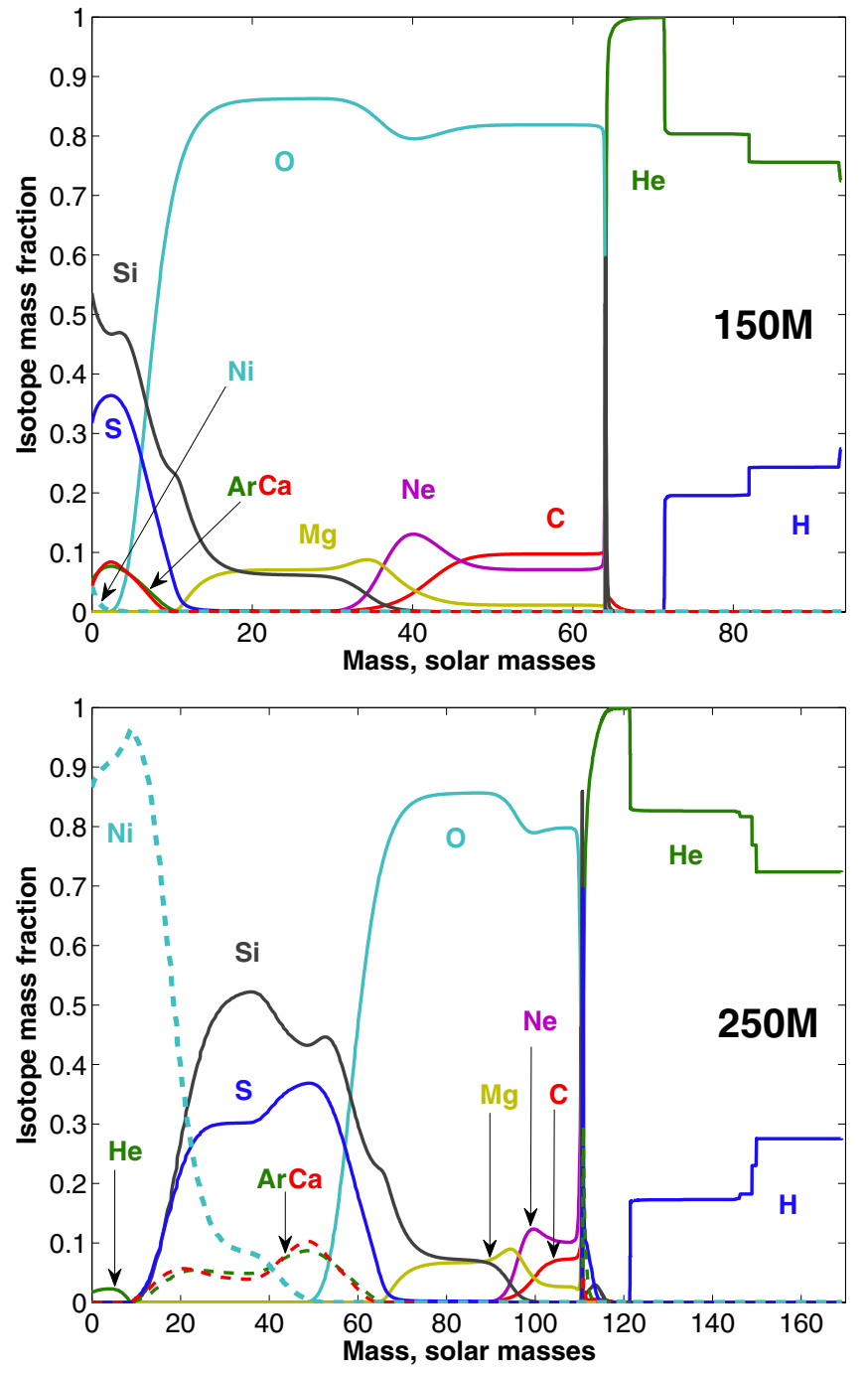

Fig. 4. Final chemical structure of our models.

We summarize the chemical yields and the production factors of each isotope from our explosion models in Table 3. Here the production factor of a given isotope is defined as

$p_{\text {iso }}=\frac{m_{\text {iso }}}{X_{\text {iso }}^{\odot} \times M_{\text {tot }}}$,

where $m_{\text {iso }}$ is the total yield of a given isotope in solar masses, $X_{\text {iso }}^{\odot}$ is the mass fraction of the isotope according to the solar metallicity pattern, and $M_{\text {tot }}$ is the initial mass of the star ( $150 M_{\odot}$ or $250 M_{\odot}$ in the present study). The effect of radioactive decays is fully considered in the final set of the isotope yields that only consists of stable nuclei. 
A. Kozyreva et al.: Explosion and nucleosynthesis of low-redshift pair-instability supernovae

Table 3. Total nucleosynthetic yields in solar masses and production factors for our $150 M_{\odot}$ and $250 M_{\odot}$ models.

\begin{tabular}{|c|c|c|c|c|c|c|c|c|c|}
\hline & \multicolumn{2}{|c|}{ Yields $\left[M_{\odot}\right]$} & \multicolumn{2}{|c|}{ Prod. factor } & & \multicolumn{2}{|c|}{ Yields $\left[M_{\odot}\right]$} & \multicolumn{2}{|c|}{ Prod. factor } \\
\hline & $150 M_{\odot}$ & $250 M_{\odot}$ & $150 M_{\odot}$ & $250 M_{\odot}$ & & $150 M_{\odot}$ & $250 M_{\odot}$ & $150 M_{\odot}$ & $250 M_{\odot}$ \\
\hline${ }^{1} \mathrm{H}$ & 36.56 & 57.10 & 0.34 & 0.32 & ${ }^{41} \mathrm{~K}$ & $1.63(-4)$ & $3.15(-4)$ & 3.63 & 4.22 \\
\hline${ }^{2} \mathrm{H}$ & $7.10(-11)$ & $1.63(-10)$ & $1.71(-8)$ & $2.36(-8)$ & ${ }^{40} \mathrm{Ca}$ & 0.49 & 2.80 & 45.99 & 156.82 \\
\hline${ }^{3} \mathrm{He}$ & $5.01(-5)$ & $5.47(-5)$ & $9.80(-3)$ & $6.42(-3)$ & ${ }^{42} \mathrm{Ca}$ & $1.87(-4)$ & $2.09(-4)$ & 2.50 & 1.67 \\
\hline${ }^{4} \mathrm{He}$ & 49.16 & 81.53 & 1.20 & 1.19 & ${ }^{43} \mathrm{Ca}$ & $6.48(-8)$ & $2.07(-5)$ & $4.30(-3)$ & 0.78 \\
\hline${ }^{6} \mathrm{Li}$ & $4.93(-12)$ & $2.10(-11)$ & $4.45(-5)$ & $1.14(-4)$ & ${ }^{44} \mathrm{Ca}$ & $1.14(-4)$ & $9.65(-4)$ & 0.45 & 2.28 \\
\hline${ }^{7} \mathrm{Li}$ & $7.17(-10)$ & $4.57(-8)$ & $4.55(-4)$ & $1.74(-2)$ & ${ }^{46} \mathrm{Ca}$ & 0 & 0 & 0 & 0 \\
\hline${ }^{9} \mathrm{Be}$ & $8.77(-12)$ & $1.88(-11)$ & $3.01(-4)$ & $3.88(-4)$ & ${ }^{48} \mathrm{Ca}$ & $8.71(-20)$ & $3.92(-17)$ & $3.50(-15)$ & $9.45(-13)$ \\
\hline${ }^{10} \mathrm{~B}$ & $2.14(-10)$ & $4.25(-10)$ & $1.43(-3)$ & $1.70(-3)$ & ${ }^{45} \mathrm{Sc}$ & $4.32(-6)$ & $1.18(-5)$ & 0.64 & 1.05 \\
\hline${ }^{11} \mathrm{~B}$ & $7.17(-10)$ & $4.58(-9)$ & $1.07(-3)$ & $4.10(-3)$ & ${ }^{46} \mathrm{Ti}$ & $9.23(-5)$ & $1.14(-4)$ & 2.29 & 1.69 \\
\hline${ }^{12} \mathrm{C}$ & 2.22 & 0.90 & 6.04 & 1.46 & ${ }^{47} \mathrm{Ti}$ & $3.87(-7)$ & $2.42(-6)$ & $1.04(-2)$ & $3.92(-2)$ \\
\hline${ }^{13} \mathrm{C}$ & $3.58(-4)$ & $5.60(-4)$ & $8.00(-2)$ & $7.52(-2)$ & ${ }^{48} \mathrm{Ti}$ & $2.02(-4)$ & $1.69(-2)$ & 0.54 & 26.96 \\
\hline${ }^{14} \mathrm{~N}$ & $4.10(-2)$ & $7.27(-2)$ & 0.34 & 0.37 & ${ }^{49} \mathrm{Ti}$ & $1.91(-5)$ & $4.61(-4)$ & 0.68 & 9.81 \\
\hline${ }^{15} \mathrm{~N}$ & $1.17(-4)$ & $3.37(-5)$ & 0.25 & $4.31(-2)$ & ${ }^{50} \mathrm{Ti}$ & $4.23(-12)$ & $3.35(-12)$ & $1.53(-7)$ & $7.29(-8)$ \\
\hline${ }^{16} \mathrm{O}$ & 46.86 & 41.96 & 47.33 & 25.43 & ${ }^{50} \mathrm{~V}$ & $1.63(-10)$ & $1.65(-10)$ & $1.04(-3)$ & $6.28(-4)$ \\
\hline${ }^{17} \mathrm{O}$ & $1.10(-4)$ & $1.54(-4)$ & 0.28 & 0.24 & ${ }^{51} \mathrm{~V}$ & $1.93(-5)$ & $6.42(-4)$ & 0.30 & 5.98 \\
\hline${ }^{18} \mathrm{O}$ & $1.24(-4)$ & $4.94(-5)$ & 5.57 & $1.33(-2)$ & ${ }^{50} \mathrm{Cr}$ & $2.43(-4)$ & $1.02(-3)$ & 1.98 & 4.99 \\
\hline${ }^{19} \mathrm{~F}$ & $3.76(-7)$ & $4.03(-7)$ & 5.36 & $3.45(-3)$ & ${ }^{52} \mathrm{Cr}$ & $1.62(-3)$ & 0.38 & 0.66 & 91.77 \\
\hline${ }^{20} \mathrm{Ne}$ & 2.58 & 1.85 & 14.69 & 6.32 & ${ }^{53} \mathrm{Cr}$ & $1.79(-4)$ & $1.40(-2)$ & 0.63 & 29.60 \\
\hline${ }^{21} \mathrm{Ne}$ & $4.17(-4)$ & $8.79(-5)$ & 0.95 & 0.12 & ${ }^{54} \mathrm{Cr}$ & $1.09(-8)$ & $2.02(-8)$ & $1.51(-4)$ & $1.69(-4)$ \\
\hline${ }^{22} \mathrm{Ne}$ & $1.19(-3)$ & $1.17(-3)$ & $8.42(-2)$ & $4.94(-2)$ & ${ }^{55} \mathrm{Mn}$ & $1.00(-3)$ & $5.73(-2)$ & 0.45 & 15.59 \\
\hline${ }^{23} \mathrm{Na}$ & $1.39(-2)$ & $8.67(-3)$ & 2.39 & 0.90 & ${ }^{54} \mathrm{Fe}$ & $2.12(-2)$ & 0.21 & 1.82 & 11.07 \\
\hline${ }^{24} \mathrm{Mg}$ & 2.30 & 2.52 & 27.12 & 17.83 & ${ }^{56} \mathrm{Fe}$ & $4.62(-2)$ & 19.33 & 0.24 & 61.36 \\
\hline${ }^{25} \mathrm{Mg}$ & $1.34(-2)$ & $5.37(-3)$ & 1.20 & 0.29 & ${ }^{57} \mathrm{Fe}$ & $4.41(-4)$ & 0.21 & $9.93(-2)$ & 28.38 \\
\hline${ }^{26} \mathrm{Mg}$ & $2.68(-2)$ & $1.11(-2)$ & 2.09 & 0.52 & ${ }^{58} \mathrm{Fe}$ & $4.09(-6)$ & $5.44(-5)$ & $6.81(-3)$ & $5.44(-2)$ \\
\hline${ }^{27} \mathrm{Al}$ & $1.14(-2)$ & $4.20(-2)$ & 1.15 & 2.53 & ${ }^{59} \mathrm{Co}$ & $3.99(-5)$ & $5.11(-3)$ & $6.63(-2)$ & 5.10 \\
\hline${ }^{28} \mathrm{Si}$ & 6.17 & 23.08 & 54.52 & 122.29 & ${ }^{58} \mathrm{Ni}$ & $1.83(-3)$ & 0.37 & 0.22 & 26.73 \\
\hline${ }^{29} \mathrm{Si}$ & $4.62(-2)$ & $3.24(-2)$ & 7.76 & 3.26 & ${ }^{60} \mathrm{Ni}$ & $1.24(-4)$ & $7.35(-2)$ & $3.75(-2)$ & 13.36 \\
\hline${ }^{30} \mathrm{Si}$ & $2.52(-2)$ & $1.35(-2)$ & 6.19 & 2.00 & ${ }^{61} \mathrm{Ni}$ & $2.57(-7)$ & $3.75(-3)$ & $1.76(-3)$ & 15.44 \\
\hline${ }^{31} \mathrm{P}$ & $2.96(-3)$ & $8.16(-3)$ & 2.60 & 4.30 & ${ }^{62} \mathrm{Ni}$ & $9.40(-7)$ & $2.59(-2)$ & $1.99(-3)$ & 32.86 \\
\hline${ }^{32} \mathrm{~S}$ & 2.82 & 14.32 & 47.47 & 144.69 & ${ }^{64} \mathrm{Ni}$ & $7.44(-12)$ & $4.46(-9)$ & $6.00(-8)$ & $2.16(-5)$ \\
\hline${ }^{33} \mathrm{~S}$ & $3.83(-3)$ & $8.14(-3)$ & 7.93 & 10.11 & ${ }^{63} \mathrm{Cu}$ & $3.95(-9)$ & $2.40(-5)$ & $3.92(-5)$ & 0.14 \\
\hline${ }^{34} \mathrm{~S}$ & $8.17(-3)$ & $1.12(-2)$ & 2.93 & 2.41 & ${ }^{65} \mathrm{Cu}$ & $1.76(-11)$ & $9.92(-6)$ & $3.80(-7)$ & 0.13 \\
\hline${ }^{36} \mathrm{~S}$ & $2.36(-8)$ & $5.23(-8)$ & $1.96(-3)$ & $2.62(-3)$ & ${ }^{64} \mathrm{Zn}$ & $1.98(-9)$ & $1.20(-4)$ & $1.18(-5)$ & 0.43 \\
\hline${ }^{35} \mathrm{Cl}$ & $7.24(-4)$ & $1.09(-2)$ & 1.19 & 10.70 & ${ }^{66} \mathrm{Zn}$ & $6.34(-11)$ & $2.24(-4)$ & $6.40(-7)$ & 1.36 \\
\hline${ }^{37} \mathrm{Cl}$ & $6.68(-4)$ & $1.37(-3)$ & 3.25 & 4.01 & ${ }^{67} \mathrm{Zn}$ & $5.63(-15)$ & $1.56(-7)$ & $3.81(-10)$ & $6.34(-3)$ \\
\hline${ }^{36} \mathrm{Ar}$ & 0.51 & 2.93 & 37.28 & 128.15 & ${ }^{68} \mathrm{Zn}$ & $8.90(-15)$ & $4.64(-8)$ & $1.30(-10)$ & $4.06(-4)$ \\
\hline${ }^{38} \mathrm{Ar}$ & $6.75(-3)$ & $7.92(-3)$ & 2.57 & 1.81 & ${ }^{70} \mathrm{Zn}$ & 0 & 0 & 0 & 0 \\
\hline${ }^{40} \mathrm{Ar}$ & $1.37(-10)$ & $3.11(-9)$ & $3.26(-5)$ & $4.42(-4)$ & ${ }^{69} \mathrm{Ga}$ & $4.22(-16)$ & $4.47(-13)$ & $6.45(-11)$ & $4.10(-8)$ \\
\hline${ }^{39} \mathrm{~K}$ & $1.23(-3)$ & $1.16(-2)$ & 2.09 & 11.82 & ${ }^{71} \mathrm{Ga}$ & 0 & 0 & 0 & 0 \\
\hline${ }^{40} \mathrm{~K}$ & $3.71(-8)$ & $1.68(-6)$ & $3.95(-2)$ & 1.07 & ${ }^{70} \mathrm{Ge}$ & $6.93(-16)$ & $9.16(-15)$ & $8.82(-11)$ & $7.00(-10)$ \\
\hline
\end{tabular}

Notes. Yields include matter lost by the stellar wind and decay products.

We used the solar abundances taken from Woosley \& Weaver (1995), which were adopted from Anders \& Grevesse (1989) to be consistent in our comparison to other PISN and CCSN nucleosynthetic yields. A more recent study of solar abundances (Asplund et al. 2009) shows somewhat different solar abundances. The overall fraction of heavy elements differs by a factor of 0.7 mostly because of a reduced oxygen abundance. $Z=0.0134$ in Asplund et al. (2009) and $Z=0.0201$ in Anders $\&$ Grevesse (1989). However, the solar abundances serve as a denominator for our qualitative comparison. Generally, the relative scatter of the elemental/isotopic production factor (i.e. oddeven effect) remains the same. We plot all production factors in Figs. 6, 7, and 9 in logarithmic scale. Therefore, using the lower metal fraction will shift all data (except for hydrogen and helium) by $|\log 0.7| \simeq 0.15$ dex.

The pair-instability explosion in our models is mostly driven by oxygen burning because oxygen is the most abundant element at core carbon exhaustion. Our models $150 \mathrm{M}$ and $250 \mathrm{M}$ contain $64 M_{\odot}$ and $110 M_{\odot}$ oxygen cores, correspondingly, and a large portion of the oxygen core remains unburned: more than $40 M_{\odot}$ of oxygen enrich the circumstellar medium, making oxygen the third-most abundant element after hydrogen and helium. Note that only about $3 M_{\odot}$ of oxygen are produced in an ordinary core-collapse SN (Woosley \& Weaver 1995) and even less (about $0.1 M_{\odot}$ ) is left after a SN Ia (Iwamoto et al. 1999; Travaglio et al. 2004).

The highest yields in model $150 \mathrm{M}$ are those of intermediate even-charged isotopes between oxygen and sulphur $\left(2.6 M_{\odot}\right.$ of ${ }^{20} \mathrm{Ne}, 2.3 M_{\odot}$ of ${ }^{24} \mathrm{Mg}, 6.2 M_{\odot}$ of ${ }^{28} \mathrm{Si}, 2.8 M_{\odot}$ of $\left.{ }^{32} \mathrm{~S}\right)$ because only a small fraction of silicon is burned in this PISN (see Table 3). The yields of iron-group isotopes are fairly low compared with intermediate-mass isotopes. At the same time, the ejecta of model $250 \mathrm{M}$ contain large amounts of intermediatemass isotopes similar to model $150 \mathrm{M}\left(1.85 M_{\odot}\right.$ of ${ }^{20} \mathrm{Ne}, 2.5 M_{\odot}$ of ${ }^{24} \mathrm{Mg}, 23.1 M_{\odot}$ of ${ }^{28} \mathrm{Si}, 14.3 M_{\odot}$ of ${ }^{32} \mathrm{~S}, 2.9 M_{\odot}$ of ${ }^{36} \mathrm{Ar}$, $2.8 M_{\odot}$ of ${ }^{40} \mathrm{Ca}$ ), significant amounts of iron-group isotopes $\left(0.4 M_{\odot}\right.$ of ${ }^{52} \mathrm{Cr}, 0.2 M_{\odot}$ of ${ }^{54} \mathrm{Fe}, 0.2 M_{\odot}$ of ${ }^{57} \mathrm{Fe}, 0.4 M_{\odot}$ of $\left.{ }^{58} \mathrm{Ni}\right)$, and a very high iron-56 yield $\left(19.3 M_{\odot}\right)$. There is a gap around 
the titanium isotopes since these are the bottle-neck isotopes between QSE-groups (Hix \& Thielemann 1996).

Large amounts of silicon ( $6 M_{\odot}$ and $23 M_{\odot}$, respectively) are left after incomplete silicon burning in both models, which are 10-100 times higher than silicon yields resulting from corecollapse $\mathrm{SNe}$ and $\mathrm{SNe}$ Ia. The average yield of silicon in corecollapse SNe and SNe Ia is $0.4 M_{\odot}$ and $0.6 M_{\odot}$, respectively. A large amount of radioactive nickel produced in our higher mass model 250M $\left(19.3 M_{\odot}\right)$ will result in a very bright and broad supernova light curve (Scannapieco et al. 2005; Kasen et al. 2011; Kozyreva et al. 2014). This is much higher than the average iron yield of $0.04-0.2 M_{\odot}$ in core-collapse $\mathrm{SNe}$ and $0.5 M_{\odot}$ in SNe Ia (Patat et al. 1994; Smartt 2009).

We emphasize here that neither of our PISN models produces high amounts of isotopes beyond the iron-group: the mass fractions of copper, zinc gallium, and germanium isotopes are well below $10^{-4}$. The lack of $r$ - and $s$-isotopes is explained mostly by the neutron deficiency that is explained below ${ }^{3}$.

Pair-instability SNe occur because the cores of their progenitors remain much less dense than those of core-collapse SN progenitors (cf. Langer 2012). This makes neutronization during the final evolutionary stages much less significant than in core-collapse progenitors. Heger \& Woosley (2002) showed that this results in a remarkable deficiency of odd-charged nuclei compared to even-charged nuclei in the nucleosynthesis of Population III PISNe. Table 1 shows that our starting models have much higher neutron excesses $\left(\eta_{\mathrm{i}} \sim 10^{-4}\right)$ than the initial Population III star models of Heger \& Woosley $\left(\eta_{\mathrm{i}} \sim 10^{-7}\right)$. However, the neutron excess in the central region $\left(\eta_{\mathrm{c}}\right)$ where silicon burning occurs does not increase much. The highest neutron excess achieved at the centre $\left(\eta_{\mathrm{c}}^{\max }\right)$ in the $150 M_{\odot}$ and $250 M_{\odot}$ models is only $2 \times 10^{-4}$ and $1.6 \times 10^{-3}$. In the comparable Population III helium star models of Heger \& Woosley (2002; i.e. their $70 M_{\odot}$ and $115 M_{\odot}$ models; see Fig. 2), the values of $\eta_{\mathrm{c}}^{\max }$ are $2.8 \times 10^{-4}$ and $7.3 \times 10^{-4}$ (Table 1 ), which is a factor of $1.4 \ldots 2.2$ lower.

This indicates that the neutron excess in PISNe during the explosion phase does not increase by much more than about $10^{-3}$ compared with the values at core helium exhaustion. Interestingly, this implies that even if the initial metallicity of a PISN progenitor was as high as $Z_{\odot}$, the neutron excess in the core would not become much higher than about $10^{-3}$, which is the typical value of the neutron excess in the innermost layers of solar metallicity massive stars at core helium exhaustion. This value is much lower than the neutron excess achieved in the silicon shell of a typical core-collapse progenitor $\left(\eta \cong 10^{-2}\right)$, where explosive nuclear burning occurs during the supernova explosion. We conclude that the odd-even effect is expected to be significant even in metal-rich PISNe.

Heger \& Woosley (2005) argued that an unusually strong mixing of nitrogen into the helium core of a PISN progenitor may make the odd-even effect as weak as in the nucleosynthesis of a core-collapse supernova. However, to increase the neutron excess to $\eta \cong 10^{-2}$ by such mixing is very difficult to achieve in massive stars, as discussed in Yoon et al. (2012). It requires abundant production of primary nitrogen in the first place, which in turn requires efficient chemical mixing of carbon and oxygen into hydrogen-shell burning during the post-main sequence phases. Then, the primary nitrogen has to be mixed into the core of the star to increase the neutron excess, via the ${ }^{14} \mathrm{~N}(\alpha, \gamma){ }^{18} \mathrm{~F}\left(\mathrm{e}^{+}, v\right){ }^{18} \mathrm{O}$ reaction. One possibility for such mixing

\footnotetext{
3 Note that in the nuclear network used in our study no element heavier than germanium is included.
}

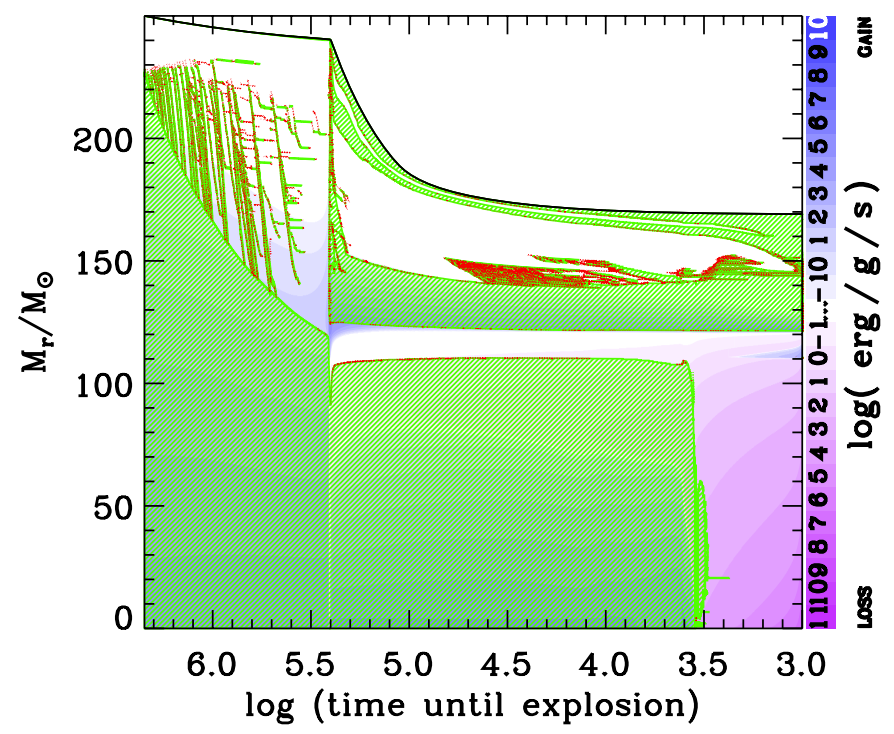

Fig. 5. Kippenhahn diagram for the $250 M_{\odot}$ PISN model. Convective and semi-convective layers are marked by green hatched lines and red dots. The net amount of local energy loss and production is indicated by colour shading. The surface of the star is marked by the black solid line.

is the penetration of the helium-burning convective core into the hydrogen burning shell source, which is often observed in massive Population III star models (Heger \& Woosley 2010; Yoon et al. 2012). Such mixing of nitrogen into the stellar core by convection is not observed in our PISN progenitor models, as shown in Fig. 5. Even if it occurred, it would be difficult to enhance the neutron excess to more than about $10^{-3}$ (Yoon et al. 2012). Another possibility is rotationally induced mixing. Our models are initially slow rotators and lost most of their initial angular momentum via mass loss, rendering the role of rotation unimportant. Yoon et al. (2012) concluded that mixing of nitrogen resulting from rotation may not enhance the neutron excess by more than about $10^{-5}$ even for the extreme case of the socalled chemically homogeneous evolution. Furthermore, PISNe through chemically homogeneous evolution are expected only at the extremely low metallicity of $Z \lesssim 10^{-5}$ (Yoon et al. 2006; Langer et al. 2007). We conclude that the neutron excess does not exceed $\sim 10^{-3}$ in most PISN progenitors.

As expected from the above discussion, the odd-even effect in our models also appears to be strong (Figs. 6, 7, and 9). The ratio of the even- to odd-charged isotope mass fractions reaches $10^{2}-10^{5}$, which is far from the observed values in the solar system and in metal-poor stars (Cayrel et al. 2004; Christlieb et al. 2004; Frebel et al. 2005). However, this effect is significantly weaker than in the corresponding Population III star models, for relatively light nuclei (i.e., lighter than silicon for the $150 M_{\odot}$ model and calcium for the $250 M_{\odot}$ model). This is because these elements are produced in the upper layers of the star where neutronization during the explosive phase does not occur and the degree of the odd-even effect is largely determined by the initial metallicity of the star. For example, the production factors of magnesium and sodium differ by $1.2 \mathrm{dex}$ in our $250 M_{\odot}$ model, while this difference increases to $2.1 \mathrm{dex}$ in the corresponding Population III model.

Moreover, the overall production factors of our models are smaller than those of the corresponding Population III star models even though the total yields of heavy elements are similar, as shown in Table 2. For example, the production factors of iron 

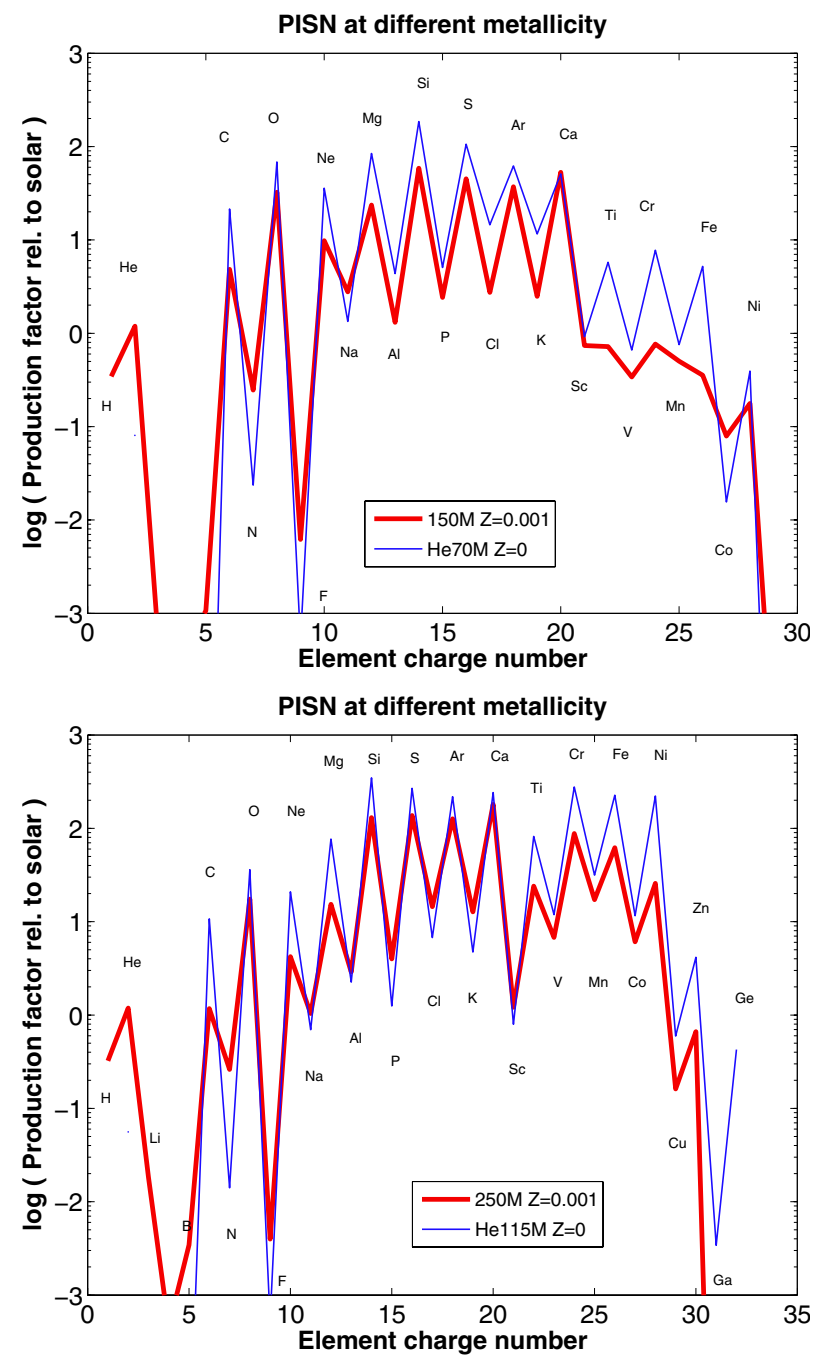

Fig. 6. Production factors of the main elements from our $150 M_{\odot}$ (upper panel) and $250 M_{\odot}$ (lower panel) PISN models (red thick lines) compared with those of the similar $70 M_{\odot}$ (upper panel) and $115 M_{\odot}$ (lower panel) Population III helium star model by Heger \& Woosley (2002) (blue thin lines).

from our $250 M_{\odot}$ model and a Population III $115 M_{\odot}$ helium star model are 59 and 125 , respectively, while both models give the same total amount of iron (about $19 M_{\odot}$ ). The reason for this difference is simply that no hydrogen envelope is included in the Population III helium star models (i.e., the helium-core masses correspond to the initial masses), while in our models the helium-core masses are only certain fractions of the initial masses.

In the next section, we discuss the implications of this result for the chemical evolution of galaxies.

\section{Implications for chemical evolution}

As mentioned above, very massive stars at relatively high metallicity are assumed to loose too much mass to produce PISNe, and metal-poor environments are preferred for PISN progenitors. Langer et al. (2007) argued that the metallicity threshold for PISNe ( $Z_{\text {PISN }}$ ) may be about $Z_{\odot} / 10 \ldots Z_{\odot} / 3$. This conclusion agrees with the recent result of another detailed numerical study by Yusof et al. (2013).
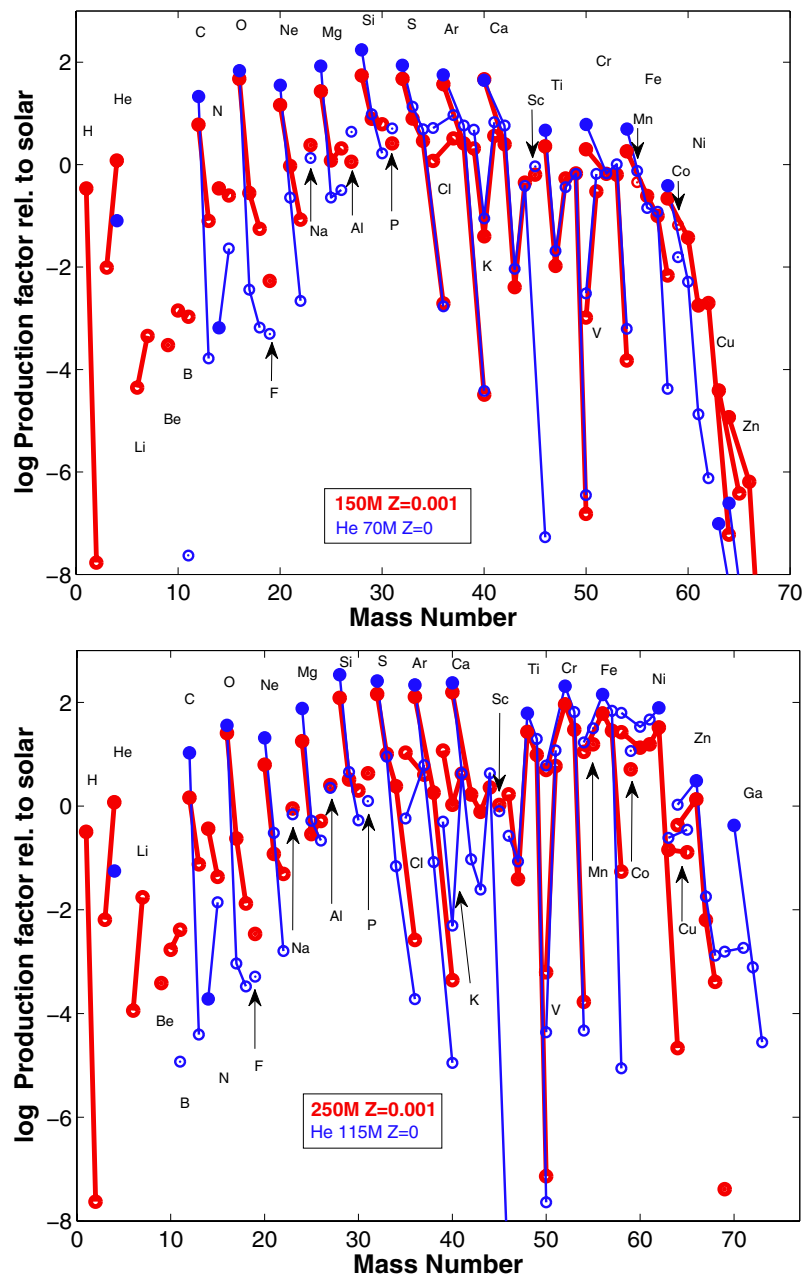

Fig. 7. Isotopic production factors for the indicated nuclei. The isotopes of a given element are connected by solid lines. The filled circles indicate the most abundant isotope for each element, while the open circles denote the other isotopes. Our $150 M_{\odot}$ (upper panel) and $250 M_{\odot}$ (lower panel) PISN models (red thick) are compared with those of the similar $70 M_{\odot}$ (upper panel) and $115 M_{\odot}$ (lower panel) Population III helium star model by Heger \& Woosley (2002) (blue thin lines).

Therefore, it is an important question how PISNe contributed to the chemical evolution of galaxies in environments with $Z \lesssim$ $Z_{\text {PISN }}$. This critically depends on how many PISN progenitors form as a function of time. There are several possibilities for the formation mechanism of very massive stars (cf. Zinnecker \& Yorke 2007), including very rapid mass accretion (e.g. Hosokawa \& Omukai 2009), mergers in close binary systems, and stellar collisions (e.g., Yungelson et al. 2008; Pan et al. 2012b). Recent observations indicate that the upper stellar mass limit $\left(M_{\mathrm{UP}}\right)$ may be as high as $180 M_{\odot}$ in our Galaxy and $300 M_{\odot}$ in the Large Magellanic Cloud (Crowther et al. 2010; Schneider et al. 2014). Because of the paucity of very massive stars discovered in the local Universe, these observations still do not give a good constraint on the initial mass function for potential progenitors of PISNe. If we simply assume a Salpeter-like IMF $(\Gamma=-2.3)$, about $2 \%$ of all supernova progenitors $\left(M \gtrsim 12 M_{\odot}\right)$ have initial masses high enough $\left(M \gtrsim 140 M_{\odot}\right)$ to produce a PISN.

To evaluate the contribution of PISNe to chemical evolution compared with that of core-collapse $\mathrm{SNe}$, we calculated the production factor of major nuclei in the following way. The 


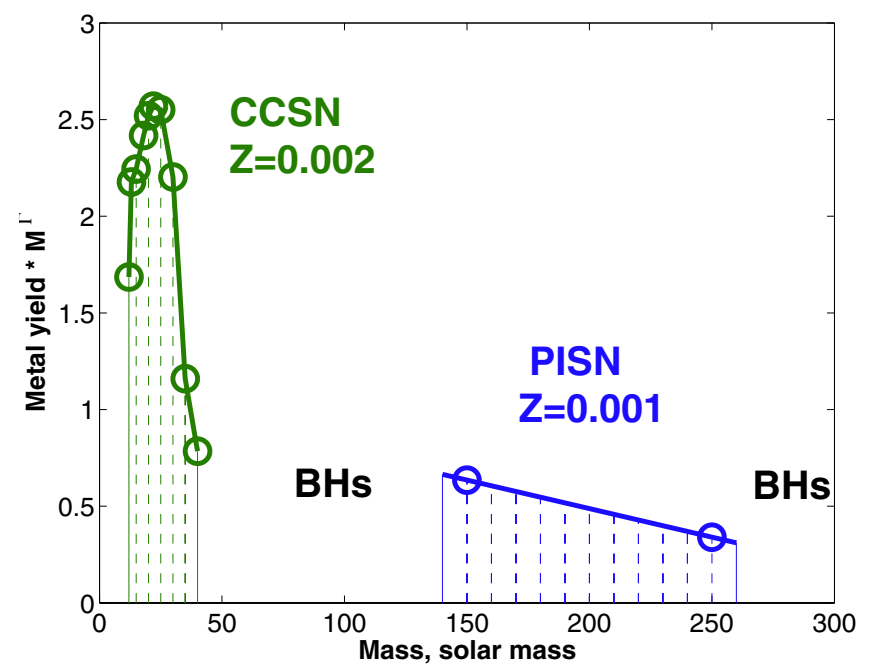

Fig. 8. Total metal yields of core-collapse $\mathrm{SN}$ models at $Z=0.002$ provided by Woosley \& Weaver (1995) (the A-series SNe models; green line) and of PISNe at $Z=0.001$ (blue line), multiplied by the initial mass function probability $\left(\Phi(M) \propto M^{\Gamma}\right)$ as a function of the initial mass. The $y$-axis is given in arbitrary units. Here, the PISN yields in the range 140-260 $M_{\odot}$ are given by the extrapolation and interpolation of our $150 M_{\odot}$ and $250 M_{\odot}$ model results. We assumed a negligible metal yield for $40 M_{\odot}<M<140 M_{\odot}$ and above $260 M_{\odot}$.

production factor integrated over an $\operatorname{IMF}\left(\Phi(M) \propto M^{\Gamma}\right)$ relative to solar abundances for a given isotope is given by

$P^{\text {int }}=\frac{\int_{12}^{260} m_{\text {iso }} \Phi(M) \mathrm{d} M}{\int_{12}^{260} X_{\text {iso }}^{\odot} M \Phi(M) \mathrm{d} M}=\frac{\int_{12}^{260} m_{\text {iso }} M^{\Gamma} \mathrm{d} M}{\int_{12}^{260} X_{\text {iso }}^{\odot} M^{\Gamma+1} \mathrm{~d} M}$.

Here, the smallest and the largest masses for supernova progenitors are assumed to be $12 M_{\odot}$ and $260 M_{\odot}$. We adopted the core-collapse SN yields from Woosley \& Weaver (1995). Since we have only two models at $150 M_{\odot}$ and $250 M_{\odot}$, we interpolated and extrapolated our results to cover the full PISN regime $\left(140-260 M_{\odot}\right)$ for this calculation, as shown in Fig. 8. From the qualitative analysis of metal-free helium PISN models (Heger \& Woosley 2002) we find that linear interpolation gives about $20 \%$ effect on weighted bulk yields, which corresponds to $0.1-0.2$ dex differences for the final bulk production factors.

This figure illustrates the contributions of core-collapse $\mathrm{SNe}$ and PISNe to the chemical enrichment. We assumed that yields from core-collapse $\mathrm{SNe}$ come from the explosions of massive stars in the mass range from $12 M_{\odot}$ to $40 M_{\odot}$. These values are taken from the low-energy explosion models of massive stars at a metallicity of $Z=0.002$ by Woosley \& Weaver (1995). The integration over the hatched regions in the figure denotes the IMF-weighted total amount of heavy elements (all elements heavier than helium) ejected by the stars from one generation. Note that even though there are significantly fewer stars in the PISN range than there are core-collapse progenitors, the total amount of heavy elements ejected from PISNe is similar to the integrated CCSN yield.

Here we assumed that stars with initial masses between $40 M_{\odot}$ and $140 M_{\odot}$ and also above $260 M_{\odot}$ do not considerably contribute to the enrichment of the surrounding medium with heavy elements. We recall that massive stars lose mass through winds, which may be enhanced in metals (e.g. carbon), and they contribute to the galactic enrichment even though they form black holes in the end. However, the stellar winds, also

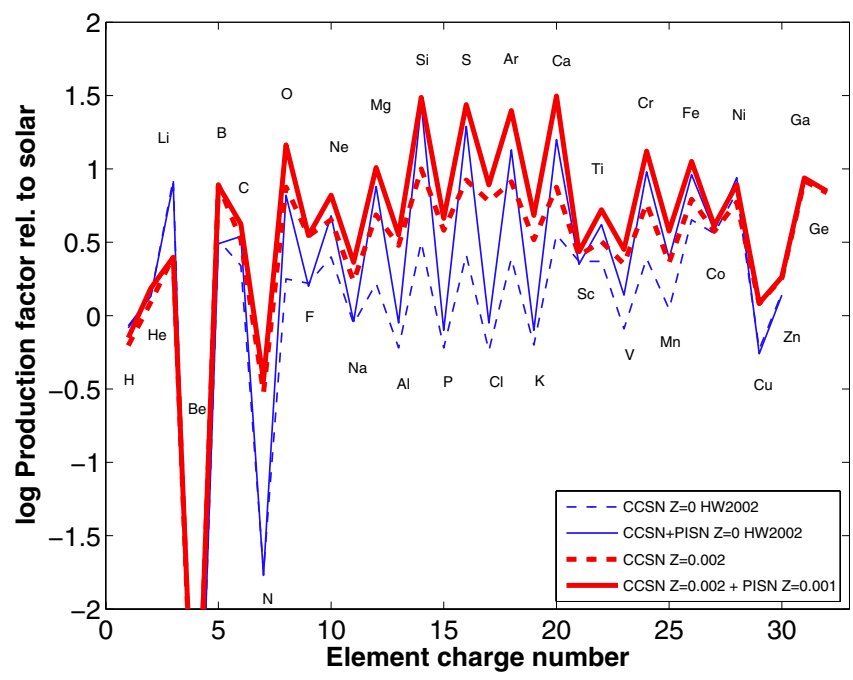

Fig. 9. Production factors relative to solar abundances of the main elements from core-collapse $\mathrm{SNe}$ (dotted lines), and from both corecollapse and pair-instability $\mathrm{SNe}$ (solid lines). The blue thin lines are the results using the Population III star models by Woosley \& Weaver (1995) and Heger \& Woosley (2002). The red thick lines correspond to the production factors using the values from Woosley \& Weaver (1995) and our study (shown in Fig. 8).

those of the carbon-rich Wolf-Rayet stars, are reduced for lower initial iron abundances (Vink \& de Koter 2005), such that their effect at the considered metallicities will be weak.

Figure 9 clearly indicates that the inclusion of PISN yields has a strong impact on the total production factors even at finite metallicity: the production factor of the even-charged nuclei is enhanced by a factor of 2-3 for most of the $\alpha$-elements with PISNe, while it is mostly negligible for the odd-charged nuclei. Note that this odd-even effect becomes strongest for the elements between Al and Sc. However, the odd-even effect is much weaker in our models than in the Population III models. The Population III yields give almost 10 times higher production factors of even-charged nuclei when PISNe are included. This difference arises mainly because the overall core-collapse SN yields at a metallicity of $Z=0.002$ are significantly higher than those of Population III core-collapse $\mathrm{SNe}$, and partly because our PISN models have a somewhat weaker odd-even effect than the Population III models, as discussed above.

As discussed in Sect. 1, the event rate of PISNe is expected to decrease to zero for metallicities higher than about $Z=Z_{\odot} / 3$. The nucleosynthetic signature of PISNe should be eliminated by the contribution of core-collapse $\mathrm{SNe}$ as the metallicity reaches the solar value, and the effect of PISNe on chemical evolution might not be found in Population I stars. However, from our study we conclude that the impact of PISNe in the environment of $Z=0.001-0.002$ may still be significant depending on the IMF, and needs to be tested in future observations of Population II stars with metallicities well below $Z=Z_{\odot} / 3$.

\section{Conclusions}

We calculated the evolution of two very massive stellar models at a metallicity of $Z=0.001$. These two models had initial zeroage main sequence masses of $150 M_{\odot}$ and $250 M_{\odot}$. The models were evolved through the core hydrogen, helium, and carbon burning (Langer et al. 2007) with the binary evolution code BEC of the Bonn stellar physics group (Yoon et al. 2006). Here, 
we continued the evolution using the same evolutionary code with an extended nuclear network where 200 isotopes are considered. We evolved these models through the electron-positron pair-creation phase and the consequent collapse and explosive oxygen and silicon burning. The $150 M_{\odot}$ and $250 M_{\odot}$ models ejected a total amount of $64 M_{\odot}$ and $111 M_{\odot}$ of metals.

An excess production of even-charged elements compared to odd-charged elements was still found in our models compared with what is found in Population III PISNe. However, the odd-even effect is weaker for most of the $\alpha$-elements than for Population III stars because of the initially higher metallicity. The nucleosynthetic pattern of the iron-group elements is critically determined by the neutronization during the explosive burning and is therefore less affected by the initial metallicity.

Given that our $150 M_{\odot}$ and $250 M_{\odot}$ models represent the low-mass and high-mass ends of PISN regime, this study allowed us to compare the PISN nucleosynthesis with that of core-collapse supernovae at a similar metallicity. The impact of PISNe on the overall nucleosynthetic pattern is expected to be weaker at $Z=0.001-0.002$ than in the metal-free environment (Fig. 9). This is mainly because of the higher initial neutron excess of our models set by higher initial metallicity.

However, the total nucleosynthetic yields from both corecollapse $\mathrm{SNe}$ and PISNe at $Z=0.001-0.002$, assuming a Salpeter IMF, would result in the enhancement of $\alpha$-elements by factors of 2-3 compared to the case without PISN contribution (Figs. 8 and 9). This analysis shows that PISNe at a metallicity of $Z=0.001$ can contribute to the enrichment of the interstellar medium with heavy elements in a similar way as Population III PISNe: the odd-even effect is still expected in metal-poor stars at metallicities of about 0.001-0.002, although its degree would be reduced by factors of 3-4 compared to the prediction from Population III stars, as shown in Fig. 9. Therefore, our models may be a useful guide for interpreting future observations of the chemical abundances in Population II stars at $Z \approx 0.001-0.002$ to constrain the number of PISNe that might have occurred in our Galaxy.

Acknowledgements. A.K. thanks Sergey Blinnikov and Alexander Heger for fruitful and helpful discussions. We also thank the referee for useful comments, which helped us to improve the draft.

\section{Appendix A: basic stellar structure equations}

The structure and evolution of stars are governed by a set of partial differential equations. The following are hydrodynamic equations that include the inertia term. However, they can easily be converted into a hydrostatic version by equating the inertia term with zero.

The set contains the equations of (1) continuity of mass; (2) momentum; and (3) energy. Written in the vector form, they are

$$
\begin{aligned}
& \frac{\partial \rho}{\partial t}+\nabla \cdot(\rho \boldsymbol{u})=0 \\
& \frac{\partial \rho \boldsymbol{u}}{\partial t}+\nabla \cdot(\boldsymbol{u} \otimes \rho \boldsymbol{u})+\nabla p_{\mathrm{g}}=0, \\
& \frac{\partial E}{\partial t}+\nabla \cdot\left[\boldsymbol{u}\left(E+p_{\mathrm{g}}\right)\right]=0,
\end{aligned}
$$

where $\rho$ is the gas density, $\boldsymbol{u}$ its velocity, $p_{\mathrm{g}}$ the gas pressure, and $E$ the total gas energy per unit volume, $E=\rho e_{\text {int }}+\frac{1}{2} \rho u^{2}$, where $e_{\text {int }}$ is the internal energy per unit mass. $\otimes$ denotes the outer product of two vectors giving a $3 \times 3$ tensor.
For a non-rotating spherically symmetric star it is appropriate to use comoving Lagrangian coordinates bound with the matter. Therefore, the above-written set of equations can be replaced by the following set:

$$
\begin{aligned}
& \left(\frac{\partial r}{\partial m}\right)_{t}=\frac{1}{4 \pi r^{2} \rho} \\
& \left(\frac{\partial r}{\partial t}\right)_{m}=u \\
& \left(\frac{\partial P}{\partial m}\right)_{t}=\frac{G m}{4 \pi r^{4}}-\frac{1}{4 \pi r^{2}}\left(\frac{\partial u}{\partial t}\right)_{m} \\
& \left(\frac{\partial l}{\partial m}\right)_{t}=\varepsilon_{\mathrm{nuc}}+\varepsilon_{v}+\frac{P}{\rho^{2}}\left(\frac{\partial \rho}{\partial t}\right)_{m}-\left(\frac{\partial \varepsilon}{\partial t}\right)_{m} \\
& \left(\frac{\partial T}{\partial m}\right)_{t}=-\nabla \frac{G m T}{4 \pi r^{4} P}\left[1+\frac{r^{2}}{G m}\left(\frac{\partial u}{\partial t}\right)_{m}\right]
\end{aligned}
$$

where $r$ is the radial distance of the shell to the centre of the star, $m$ is the mass contained - serves as mass coordinate of the shell $m(r)=\int_{0}^{r} 4 \pi r^{2} \rho \mathrm{d} r, \rho$ is the density in the shell, $u$ is the radial velocity, $P$ is the pressure, $G$ is the gravitational constant, $l$ is the local luminosity, $T$ is the temperature, $\varepsilon$ is the internal energy per unit mass, $\varepsilon_{\text {nuc }}$ corresponds to the energy release due to thermonuclear burning, and $\varepsilon_{v}$ represents the local heat losses due to neutrino flux.

The details about this set of equations can be found for example in Kippenhahn \& Weigert (1990); Heger (1998).

The temperature gradient in radiative mass shells

$$
\nabla \equiv\left(\frac{\partial \ln T}{\partial \ln P}\right)_{t}
$$

is given by radiative temperature gradient

$$
\nabla_{\mathrm{rad}}=\frac{3}{16 \pi a c G} \frac{x l P}{m T^{4}}\left[1+\frac{r^{2}}{G m}\left(\frac{\partial u}{\partial t}\right)_{m}\right]^{-1},
$$

where $a$ is the radiation constant, $c$ is the speed of light, and $\varkappa$ is the Rosseland mean opacity. The opacities are based on Alexander \& Ferguson (1994) and Iglesias \& Rogers (1996).

The temperature gradient in convective shells is calculated using the mixing-length theory (see e.g. Kippenhahn \& Weigert 1990, and references therein).

The equation of state needs to be added to this set of equations, which binds the pressure $P$ with the temperature $T$.

The set of equations is the system of non-linear partial differential equations. The evolution code solves it using the Newton-Raphson iteration method. The details about the numerical method can be found in Henyey et al. $(1959,1964)$.

\section{References}

Alexander, D. R., \& Ferguson, J. W. 1994, ApJ, 437, 879

Anders, E., \& Grevesse, N. 1989, Geochim. Cosmochim. Acta, 53, 197 Appenzeller, I. 1970, A\&A, 9, 216

Asplund, M., Grevesse, N., Sauval, A. J., \& Scott, P. 2009, ARA\&A, 47, 481

Barkat, Z., Rakavy, G., \& Sack, N. 1967, Phys. Rev. Lett., 18, 379

Bisnovatyi-Kogan, G. S., \& Kazhdan, Y. M. 1967, Sov. Ast., 10, 604

Blinnikov, S. I., Dunina-Barkovskaya, N. V., \& Nadyozhin, D. K. 1996, ApJS, 106, 171

Bodansky, D., Clayton, D. D., \& Fowler, W. A. 1968, ApJS, 16, 299

Bond, J. R., Arnett, W. D., \& Carr, B. J. 1982, in Supernovae: A Survey of Current Research, eds. M. J. Rees, \& R. J. Stoneham, NATO ASIC Proc., 90, 303

Cayrel, R., Depagne, E., Spite, M., et al. 2004, A\&A, 416, 1117 
Christlieb, N., Gustafsson, B., Korn, A. J., et al. 2004, ApJ, 603, 708 Crowther, P. A., Schnurr, O., Hirschi, R., et al. 2010, MNRAS, 408, 731 Dessart, L., Hillier, D. J., Waldman, R., Livne, E., \& Blondin, S. 2012, MNRAS, 426, L76

Dessart, L., Waldman, R., Livne, E., Hillier, D. J., \& Blondin, S. 2013, MNRAS, 428, 3227

Ekström, S., Georgy, C., Eggenberger, P., et al. 2012, A\&A, 537, A146

El Eid, M. F., \& Langer, N. 1986, A\&A, 167, 274

El Eid, M. F., Fricke, K. J., \& Ober, W. W. 1983, A\&A, 119, 54

Fowler, W. A., \& Hoyle, F. 1964, ApJS, 9, 201

Fraley, G. S. 1968, Ap\&SS, 2, 96

Frebel, A., Aoki, W., Christlieb, N., et al. 2005, Nature, 434, 871

Fuller, G. M., Fowler, W. A., \& Newman, M. J. 1982, ApJS, 48, 279

Gal-Yam, A. 2012, Science, 337, 927

Gal-Yam, A., Mazzali, P., Ofek, E. O., et al. 2009, Nature, 462, 624

Heger, A. 1998, Ph.D. Thesis, Ph.D. Dissertation, Max-Planck-Institut für Astrophysik, unpublished

Heger, A., \& Woosley, S. E. 2002, ApJ, 567, 532

Heger, A., \& Woosley, S. 2005, in From Lithium to Uranium: Elemental Tracers of Early Cosmic Evolution, eds. V. Hill, P. Francois, \& F. Primas, IAU Symp., 228, 297

Heger, A., \& Woosley, S. E. 2010, ApJ, 724, 341

Heger, A., Jeannin, L., Langer, N., \& Baraffe, I. 1997, A\&A, 327, 224

Heger, A., Langer, N., \& Woosley, S. E. 2000, ApJ, 528, 368

Heger, A., Fryer, C. L., Woosley, S. E., Langer, N., \& Hartmann, D. H. 2003, ApJ, 591, 288

Henyey, L. G., Wilets, L., Böhm, K. H., Lelevier, R., \& Levee, R. D. 1959, ApJ, 129,628

Henyey, L. G., Forbes, J. E., \& Gould, N. L. 1964, ApJ, 139, 306

Herzig, K., El Eid, M. F., Fricke, K. J., \& Langer, N. 1990, A\&A, 233, 462

Hix, W. R., \& Thielemann, F.-K. 1996, ApJ, 460, 869

Hosokawa, T., \& Omukai, K. 2009, ApJ, 703, 1810

Iglesias, C. A., \& Rogers, F. J. 1996, ApJ, 464, 943

Iwamoto, K., Brachwitz, F., Nomoto, K., et al. 1999, ApJS, 125, 439

Kasen, D., \& Bildsten, L. 2010, ApJ, 717, 245

Kasen, D., Woosley, S. E., \& Heger, A. 2011, ApJ, 734, 102

Kippenhahn, R., \& Weigert, A. 1990, Stellar Structure and Evolution, Astronomy and Astrophysics Library (Berlin: Springer-Verlag), 468

Kozyreva, A., Blinnikov, S., Langer, N., \& Yoon, S.-C. 2014, A\&A, 565, A70

Langer, N. 1991, A\&A, 252, 669
Langer, N. 2012, ARA\&A, 50, 107

Langer, N., \& El Eid, M. F. 1986, A\&A, 167, 265

Langer, N., Fricke, K. J., \& Sugimoto, D. 1983, A\&A, 126, 207

Langer, N., Norman, C. A., de Koter, A., et al. 2007, A\&A, 475, L19

Moriya, T., Tominaga, N., Tanaka, M., Maeda, K., \& Nomoto, K. 2010, ApJ, 717, L83

Nomoto, K., \& Hashimoto, M. 1988, Phys. Rep., 163, 13

Pan, T., Kasen, D., \& Loeb, A. 2012a, MNRAS, 422, 2701

Pan, T., Loeb, A., \& Kasen, D. 2012b, MNRAS, 423, 2203

Patat, F., Barbon, R., Cappellaro, E., \& Turatto, M. 1994, A\&A, 282, 731

Rakavy, G., \& Shaviv, G. 1967, ApJ, 148, 803

Scannapieco, E., Madau, P., Woosley, S., Heger, A., \& Ferrara, A. 2005, ApJ, 633, 1031

Schneider, F. R. N., Izzard, R. G., de Mink, S. E., et al. 2014, ApJ, 780, 117

Smartt, S. J. 2009, ARA\&A, 47, 63

Timmes, F. X. 1998, http://cococubed.asu.edu/code_pages/net_ torch.shtml

Timmes, F. X. 1999, ApJS, 124, 241

Travaglio, C., Hillebrandt, W., Reinecke, M., \& Thielemann, F.-K. 2004, A\&A, 425,1029

Umeda, H., \& Nomoto, K. 2002, ApJ, 565, 385

Vink, J. S., \& de Koter, A. 2005, A\&A, 442, 587

Vink, J. S., Muijres, L. E., Anthonisse, B., et al. 2011, A\&A, 531, A132

Wallerstein, G., Iben, Jr., I., Parker, P., et al. 1997, Rev. Mod. Phys., 69, 995

Whalen, D. J., Even, W., Frey, L. H., et al. 2013a, ApJ, 777, 110

Whalen, D. J., Even, W., Smidt, J., et al. 2013b, ApJ, submitted [arXiv: 1312.5360]

Woosley, S. E., \& Weaver, T. A. 1982, in Supernovae: A Survey of Current Research, eds. M. J. Rees, \& R. J. Stoneham, NATO ASIC Proc. 90, 79

Woosley, S. E., \& Weaver, T. A. 1995, ApJS, 101, 181

Yoon, S.-C., \& Cantiello, M. 2010, ApJ, 717, L62

Yoon, S.-C., \& Langer, N. 2005, A\&A, 443, 643

Yoon, S.-C., Langer, N., \& Norman, C. 2006, A\&A, 460, 199

Yoon, S.-C., Dierks, A., \& Langer, N. 2012, A\&A, 542, A113

Yungelson, L. R., van den Heuvel, E. P. J., Vink, J. S., Portegies Zwart, S. F., \& de Koter, A. 2008, A\&A, 477, 223

Yusof, N., Hirschi, R., Meynet, G., et al. 2013, MNRAS, 433, 1114

Zeldovich, Y. B., \& Novikov, I. D. 1971, in Relativistic astrophysics. Vol.1: Stars and relativity (Chicago: University of Chicago Press), eds. K.S. Thorne, \& W.D. Arnett, 522

Zinnecker, H., \& Yorke, H. W. 2007, ARA\&A, 45, 481 